\title{
Fabrication and Characterization of an Extended-Gate AlGaN/GaN-Based Heterostructure Field-Effect Transistor-Type Biosensor for Detecting Immobilized Streptavidin-Biotin Protein Complexes
}

\author{
Hee Ho Lee, Myunghan Bae, Sung-Hyun Jo, Jang-Kyoo Shin*, \\ Dong Hyeok Son, Chul-Ho Won and Jung-Hee Lee \\ School of Electronics Engineering, Kyunpook National University, \\ 80 Daehak-ro, Buk-gu, Daegu 702-701, Korea
}

(Received March 23, 2015; accepted June 15, 2015)

Key words: AlGaN/GaN, HFET, biosensor, extended-gate structure, streptavidin-biotin complexes

In this study, we fabricated an $\mathrm{AlGaN} / \mathrm{GaN}$-based heterostructure field-effect transistor (HFET)-type biosensor with an extended-gate structure for detecting streptavidin-biotin complexes. The gate voltage of the fabricated device was applied using a commercial $\mathrm{Ag} / \mathrm{AgCl}$ reference electrode. The binding of a self-assembled monolayer (SAM), streptavidin and biotin was detected by measuring the electrical characteristics of the fabricated biosensor. X-ray photoelectron spectroscopy (XPS) was used to verify the immobilization of the SAM on the Au-coated GaN surface. The proposed biosensor shows that biotin can be detected for a wide range of concentrations from 0.1 to $1000 \mathrm{ng} / \mathrm{ml}$. The long-term stability of both the extended-gate AlGaN/ GaN-based HFET-type and conventional AlGaN/GaN-based HFET-type biosensors was measured in phosphate-buffered saline (PBS) solution. A high sensitivity and excellent stability of the proposed biosensor has been experimentally verified.

\section{Introduction}

Recently, methods of investigating biomolecules (e.g., antibodies, peptides, and nucleotides) have been attracting increasing interest. Protein analysis is being shown to have an increasing number of applications in the detection and development of treatments for diseases. ${ }^{(1-3)}$ Typical methods used for protein analysis include mass

"Corresponding author: e-mail: jkshin@ee.knu.ac.kr 
spectroscopy, optical measurements, and electrochemical measurements. ${ }^{(4,5)}$ The most common method is optical measurement. This method, however, has a disadvantage in that it requires the use of fluorescent materials and expensive equipment. To overcome these problems, we propose an extended-gate $\mathrm{AlGaN} / \mathrm{GaN}$-based heterostructure fieldeffect transistor (HFET)-type biosensor.

The AlGaN/GaN-based heterostructure shows unique electronic properties, such as piezoelectric and spontaneous polarizations, which lead to the high mobility and high density of two-dimensional electron gas (2DEG) at the interface of the heterostructure. ${ }^{(6)}$ Also, the AlGaN/GaN-based heterostructure shows high chemical stability and mechanical hardness. The conducting channel of the AlGaN/GaN-based HFET is very close to the surface and extremely sensitive to the ambient, which should enhance detection sensitivity. The application of a certain external change under surface conditions, such as the binding of species to the gate region of the device, changes the piezoelectric induced carrier density in the channel of the HFET, which in turn alters the drain current.(7,8) Therefore, the HFET-type biosensor provides high sensitivity to immobilized biomolecules. Moreover, the HFET can be combined with an extendedgate structure for improving the performance of the FET-type biosensor. The advantages of the extended-gate based biosensor are long-term stability, the flexibility of the shape of the sensing area, and simple methods of passivation and packaging. ${ }^{(9-11)}$

In this study, we fabricate an extended-gate AlGaN/GaN-based heterostructure HFET-type biosensor for the detection of streptavidin-biotin complexes. We measure the drain current of this biosensor, which varies depending on the antibody-antigen reaction of biotin on streptavidin molecules. To confirm the immobilization of a self-assembled monolayer (SAM), we analyze the Au surface of a GaN sample by X-ray photoelectron spectroscopy (XPS). Biotin molecules could be detected down to a concentration as low as $0.1 \mathrm{ng} / \mathrm{ml}$. The long-term stability of the proposed extended-gate AlGaN/GaN-based HFET-type biosensor was compared with that of a conventional AlGaN/GaN-based HFET-type biosensor. The proposed biosensor was found to exhibit both the long-term stability of the extended-gate structure and the high sensitivity of the AlGaN/GaN-based heterostructure.

\section{Device Fabrication}

The $\mathrm{AlGaN} / \mathrm{GaN}$ heterostructure was grown by metal-organic chemical vapor deposition (MOCVD) on a sapphire substrate, which consisted of a $2-\mu \mathrm{m}$-thick resistive GaN layer, an 80-nm-thick undoped GaN layer, and a 25-nm-thick $\mathrm{Al}_{0.3} \mathrm{Ga}_{0.7} \mathrm{~N}$ layer with $30 \% \mathrm{Al}$ composition. The grown heterostructure exhibited a sheet carrier concentration of $9.0 \times 10^{12} \mathrm{~cm}^{-2}$ and a mobility of $1500 \mathrm{~cm}^{2} / \mathrm{Vs}$ at room temperature. The structure of the extended-gate $\mathrm{AlGaN} / \mathrm{GaN}$-based HFET-type biosensor is shown in Fig. 1. Mesa etching was carried out to define the active region by transformer-coupled-plasma reactive ion etching (TCP-RIE) using a $\mathrm{BCl}_{3} / \mathrm{Cl}_{2}$ gas mixture. To protect the $\mathrm{AlGaN}$ surface from a high-temperature rapid thermal process (RTP) for ohmic contact formation, a thin $\mathrm{Al}_{2} \mathrm{O}_{3}$ dielectric layer was then deposited at a rate of $0.7 \AA$ /cycle at $450{ }^{\circ} \mathrm{C}$ by plasma-enhanced atomic layer deposition (PEALD). ${ }^{(12)}$ For ohmic contact formation, the $\mathrm{Al}_{2} \mathrm{O}_{3}$ layer in the source/drain region was etched away and metal layers $(\mathrm{Si} / \mathrm{Ti} / \mathrm{Al} / \mathrm{Ni} / \mathrm{Au})$ were deposited 


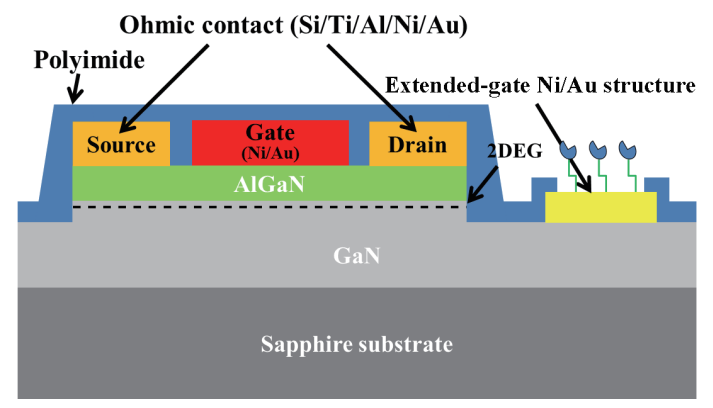

Fig. 1. (Color online) Structure of the extended-gate AlGaN/GaN-based HFET-type biosensor.

by electron beam evaporation. Then, a two-step RTP was carried out, first at a low temperature of $500{ }^{\circ} \mathrm{C}$ for $20 \mathrm{~s}$, and subsequently at a high temperature of $800{ }^{\circ} \mathrm{C}$ for 30 $\mathrm{S}$ in $\mathrm{N}_{2}$ ambient. The $\mathrm{Al}_{2} \mathrm{O}_{3}$ layer in the gate region was etched away and a metal layer $(\mathrm{Ni} /$ $\mathrm{Au}$ ) was deposited by electron beam evaporation. As shown in Fig. 1, Ni/Au was used as the gate metal because Au has a chemical affinity for thiol. SAMs on Au are stable for a period of 1-2 months in air or in contact with water. ${ }^{(13)}$ The wire bonding metal layer (Ti/Al) was deposited by electron beam evaporation and patterned by a lift-off process. Figure 2(a) shows a photograph of the fabricated biosensor. The gate length, width, and area of the fabricated AlGaN/GaN-based HFET-type biosensor are $80 \mu \mathrm{m}, 200$ $\mu \mathrm{m}$, and $250 \times 250 \mu \mathrm{m}^{2}$, respectively. After the metal deposition, a polyimide layer for passivation was coated onto the entire wafer at $2500 \mathrm{rpm}$ for $25 \mathrm{~s}$ and cured for $50 \mathrm{~min}$ at $150{ }^{\circ} \mathrm{C}$. After curing, the polyimide layer was patterned by a photolithographic process and then finally cured at $250{ }^{\circ} \mathrm{C}$ for $2 \mathrm{~h}$ to obtain a $2-\mu \mathrm{m}$-thick film. Table 1 shows the fabrication process flow for the extended-gate AlGaN/GaN-based HFET-type biosensor. Figure 2(b) shows the packaged extended-gate AlGaN/GaN-based HFET-type biosensor. To dip the fabricated sensor in the chemical solution, the sensor was attached to a printed circuit board (PCB). The fabricated chip was coated to electrically isolate it from the electrolyte using silicone rubber (3140, Dow Corning) after bonding.

\section{Measurements}

As shown in Fig. 3, we prepared a measurement system to characterize the sensor performance. A semiconductor parameter analyzer (4156, HP) was used to measure the electrical characteristics of the fabricated sensor. A commercial $\mathrm{Ag} / \mathrm{AgCl}$ reference electrode (RE-5B, BASi) was used to apply the gate bias of the fabricated sensor. To fix the activation conditions of the sample, $10 \mathrm{mM}$ phosphate-buffered saline (PBS) solution ( $\mathrm{pH} 7.4$, Gibco) was used as a background solution. Components of the buffer solution are $137 \mathrm{mM} \mathrm{NaCl}, 2.7 \mathrm{mM} \mathrm{KCl}, 10 \mathrm{mM} \mathrm{Na}_{2} \mathrm{HPO}_{4}$ and $2 \mathrm{mM} \mathrm{KH}_{2} \mathrm{PO}_{4}$. First, the drain current of the fabricated sensor was measured in the PBS solution without any biomolecules at a reference electrode voltage of $-1 \mathrm{~V}$. 


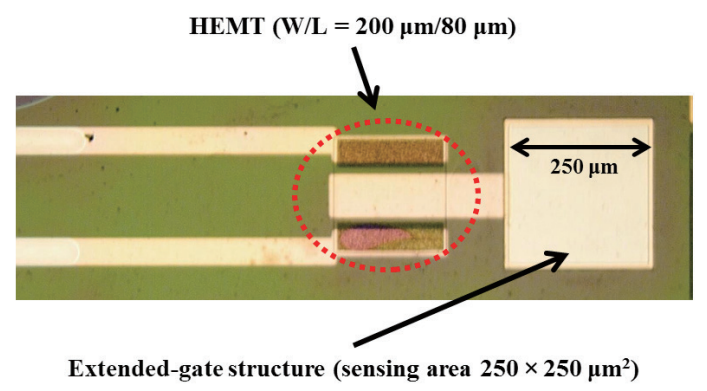

(a)

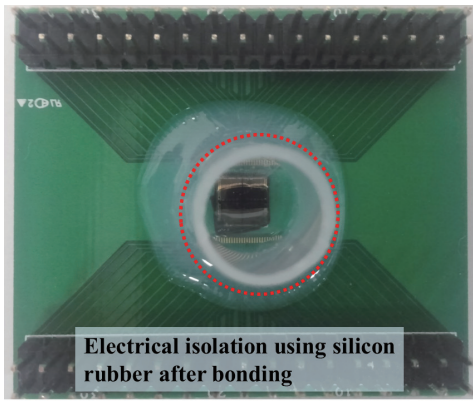

(b)

Fig. 2. (Color online) Photographs of (a) the fabricated single extended-gate AlGaN/GaN-based HFET-type biosensor $(W / L=200 \mu \mathrm{m} / 80 \mu \mathrm{m})$ and (b) the packaged extended-gate AlGaN/GaNbased HFET-type biosensor.

Table 1

Fabrication process flow for the extended-gate AlGaN/GaN-based HFET-type biosensor.

\begin{tabular}{lc}
\hline Step & Process \\
\hline 1. Wafer preparation & 105-nm-thick AlGaN/GaN heterostructure \\
2. Initial cleaning & Standard solvent and acid cleaning \\
3. Active region define & $\mathrm{TCP}-\mathrm{RIE}$ etching \\
4. Source/drain define & $\mathrm{Si} / \mathrm{Ti} / \mathrm{Al} / \mathrm{Ni} / \mathrm{Au}$ evaporation \\
5. Gate electrode deposition & $\mathrm{Ni} / \mathrm{Au}$ evaporation \\
6. Extended-gate metal deposition & $\mathrm{Ni} / \mathrm{Au}$ evaporation \\
7. Pad metal deposition & $\mathrm{Ti} / \mathrm{Al}$ evaporation \\
8. Passivation & Polyimide spin coating \\
\hline
\end{tabular}

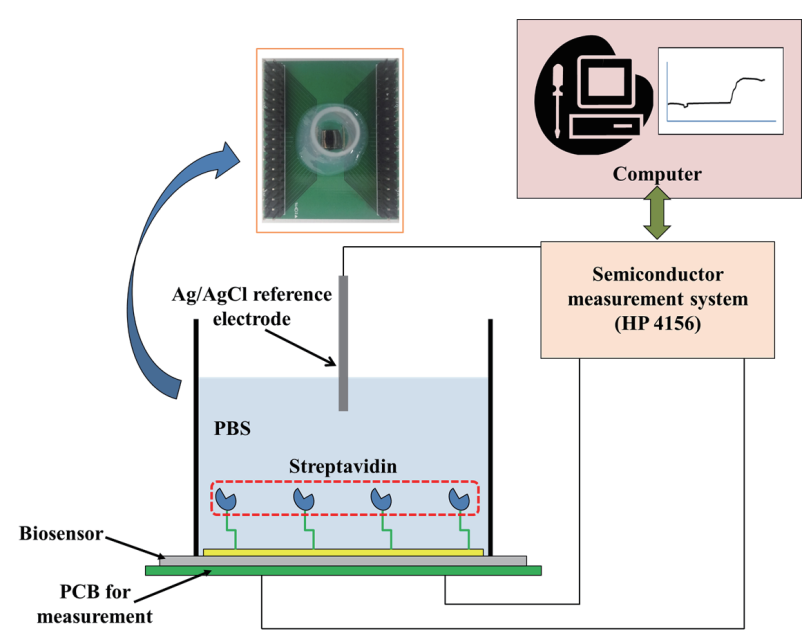

Fig. 3. (Color online) Measurement setup with the fabricated chip packaged on a designed circuit board. 
11-Mercaptoundecanoic acid (MUA) $(218.4 \mu \mathrm{l})$ (Sigma-Aldrich) was diluted in $9.7 \mathrm{ml}$ ethanol. After that, the fabricated sensor was dipped in ethanol containing 0.1 $\mathrm{mmol} / \mathrm{ml}$ diluted MUA for $12 \mathrm{~h}$. Thiols of MUA were attached to the Au surface of the extended-gate structure. According to the binding of thiols to the Au surface, a SAM was formed on the Au surface. Subsequently, the biosensor was rinsed using the new PBS solution to prevent the interference of the noncombined biomolecules in the solution. Then, the drain current of the biosensor was measured in the PBS solution. Streptavidin (1 mg) (Sigma-Aldrich) was diluted in $18.9 \mathrm{ml}$ of PBS solution. The biosensor was exposed for $12 \mathrm{~h}$ in a PBS solution containing $52.6 \mathrm{ng} / \mathrm{ml}$ streptavidin. Streptavidin can be attached to a SAM because it has an amine group. ${ }^{(9)}$ After rinsing the biosensor in a new PBS solution, the drain current was measured. Biotin was injected to investigate the interaction between streptavidin and biotin. The high-affinity streptavidin-biotin complexes are characterized by an extensive hydrogen-bonding network. ${ }^{(14)}$ Finally, the biosensor was exposed from 0.1 to $1000 \mathrm{ng} / \mathrm{ml}$ biotin individually, and for each concentration, such exposure was repeated five times to obtain the standard deviation of the drain current response.

XPS experiments were performed to verify the immobilization of MUA on the Au surface. XPS spectra were acquired using a commercial XPS system (PHI Quantera SXM) with a monochromatic Al K $\alpha$ source and a hemispherical electron energy analyzer. $\mathrm{A} \mathrm{Au} 4 \mathrm{f}$ peak at $84.0 \mathrm{eV}$ was used for data correction. The XPS measurements provide information about the chemical structure on the films and can be used to study samples prepared in the ambient environment without an additional in situ treatment. ${ }^{(13)}$

\section{Results and Discussion}

The $I-V$ electrical characteristics of the extended-gate AlGaN/GaN-based HFET-type biosensor are shown in Fig. 4. Figure 4 shows that the fabricated biosensor exhibits a depleted n-channel FET behavior. Its threshold voltage is $-4.8 \mathrm{~V}$, which is extrapolated from Fig. 4(a) in the saturation region $\left(V_{\mathrm{DS}}=7 \mathrm{~V}\right)$. The maximum drain current and maximum transconductance were measured as $10.0 \mathrm{~mA}$ and $1.91 \mathrm{mS}$, respectively.

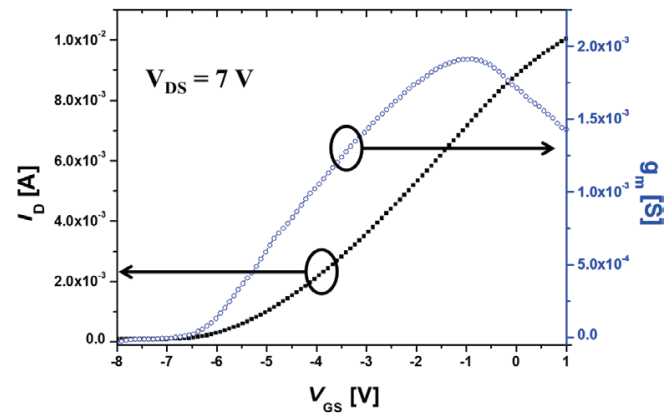

(a)

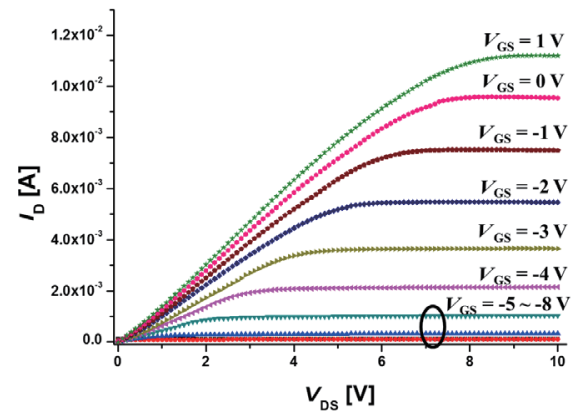

(b)

Fig. 4. (Color online) $I-V$ characteristics of the extended-gate AlGaN/GaN-based HFETbiosensor: (a) $I_{\mathrm{D}}-V_{\mathrm{GS}}$ and (b) $I_{\mathrm{D}}-V_{\mathrm{DS}}$ characteristics. 
Figure 5 shows the electrical characteristics of the extended-gate $\mathrm{AlGaN} / \mathrm{GaN}$-based HFET-type biosensor as a function of interactions among SAM, streptavidin, and biotin with the $\mathrm{Ag} / \mathrm{AgCl}$ reference electrode. At $V_{\mathrm{REF}}=-1 \mathrm{~V}$ and $V_{\mathrm{DS}}=9 \mathrm{~V}$, the drain current decreased by $1.46 \mathrm{~mA}$ because of the negative charges produced when thiol was injected into the solution and immobilized on the Au sensing gate. The net negative charges of the reactive thiol head group introduce a negative surface potential on the gate electrode of the HFET. ${ }^{(9)}$ In addition, the drain current decreased by $0.9 \mathrm{~mA}$ when streptavidin bound to the SAM, owing to the net negative charges of streptavidin. ${ }^{(7)}$ The drain current decreased by $550 \mu \mathrm{A}$ when biotin bound to streptavidin, owing to the decrease in capacitance following the binding between streptavidin and biotin. The variation in capacitance can be explained in terms of the Debye length. It is well known that only potential changes that occur within the order of the Debye length can be detected. ${ }^{(15)}$ The dimensions of macromolecules, however, such as biotin, are much larger (approximately $10 \mathrm{~nm}$ ) than those of the double layer at the electrolyte-insulator interface. Therefore, the measurable effect of potential due to the biotin charge is very small in a solution with a high ionic strength $(>0.1 \mathrm{~mol} / \mathrm{l})$ because biomolecule charges will be at a distance from the surface greater than the Debye length. ${ }^{(4)}$ Therefore, the drain current of the extended-gate $\mathrm{AlGaN} / \mathrm{GaN}$-based HFET-type biosensor decreased owing to the increase in the thickness of the capacitor layer. Consequently, variations in the drain current of the proposed biosensor can detect the conjugation of the streptavidin and biotin protein complexes.

Figure 6 shows the variation in drain voltage with biotin concentration at $V_{\mathrm{DS}}=$ $9 \mathrm{~V}$ and $V_{\mathrm{REF}}=-1 \mathrm{~V}$. From this figure, the extended-gate AlGaN/GaN-based HFETtype biosensor could be observed to detect biotin at concentrations as low as $0.1 \mathrm{ng} /$ $\mathrm{ml}$, since the $\mathrm{AlGaN} / \mathrm{GaN}$ HFET has a high electron mobility induced by piezoelectric

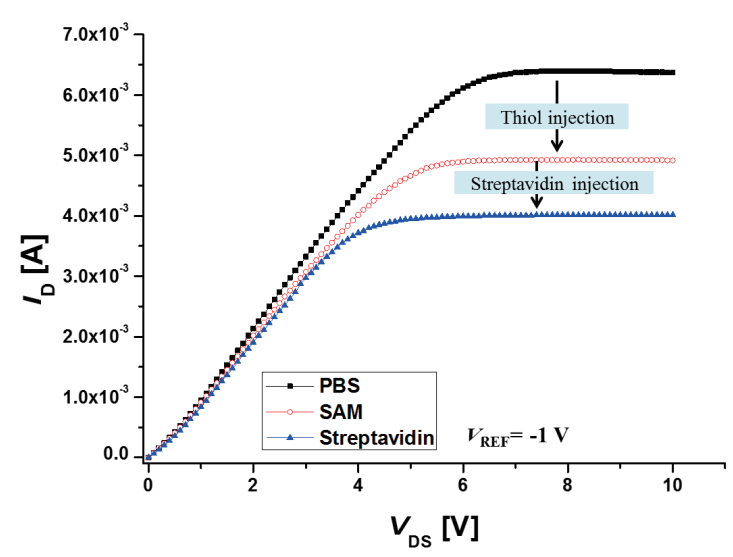

(a)

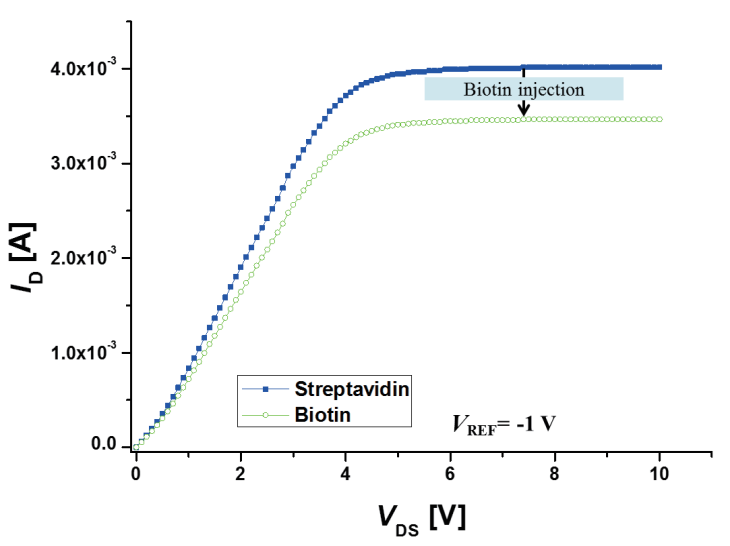

(b)

Fig. 5. (Color online) Output characteristics of the sensor with (a) SAM and streptavidin and (b) streptavidin and biotin. 


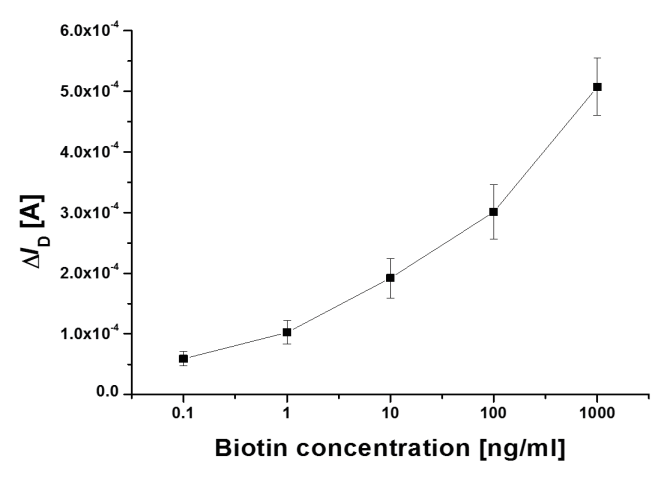

Fig. 6. Output voltage change in biosensor as a function of biotin concentration.

and spontaneous polarizations. Also, the gate region of the AlGaN/GaN HFET is very sensitive to the binding of biomolecules owing to the proximity of the 2DEG channel of the device to the surface. We could not obtain reliable results for biotin concentrations below $0.1 \mathrm{ng} / \mathrm{ml}$, and the sensitivity of the biosensor might be improved using the differential mode circuit for reducing the noise in the biosensor. ${ }^{(9)}$

Figure 7 shows the long-term stability of the extended-gate AlGaN/GaN-based HFETtype biosensor compared with that of a conventional AlGaN/GaN-based HFET-type biosensor. The long-term stability of the proposed biosensor was attributed to the good isolation between the sensor device and the measuring solution, because the measuring solution is in contact only with the extended-gate structure of the sensor device. ${ }^{(9,10)}$ For comparison, the extended-gate AlGaN/GaN-based HFET-type and conventional AlGaN/GaN-based HFET-type biosensors were fabricated by the same process, with the same device dimensions. From this figure, we confirmed that the output current of the extended-gate $\mathrm{AlGaN} / \mathrm{GaN}$-based HFET-type biosensor is more stable than that of the conventional AlGaN/GaN-based HFET-type biosensor.

To examine the SAM on the Au surface, XPS spectra were measured. A SAM is adsorbed on the $\mathrm{Ni} / \mathrm{Au}$ extended-gate via a thiol-modifying reaction, owing to a strong interaction between $\mathrm{Au}$ and the thiol group. ${ }^{(16-18)}$ To confirm that the modification was successful, XPS measurements were performed under the same conditions as the electrical measurements of the fabricated extended-gate HFET-type biosensor. Figure 8 shows the measured high-resolution XPS spectra of $S 2 p$ and $S$ 2s after SAM immobilization of the Au-coated GaN sample. The $\mathrm{S} 2 \mathrm{p}$ at binding energies of 161.3 and $160.1 \mathrm{eV}$ indicates a $\mathrm{Au}$ thiolated sulfur species. The $\mathrm{S} 2 \mathrm{~s}$ peak at $225.7 \mathrm{eV}$ was also observed for the SAM-immobilized sample. Therefore, the results of the XPS measurements support the SAM formation on the Ni/Au extended-gate of the fabricated HFET-type biosensor. 


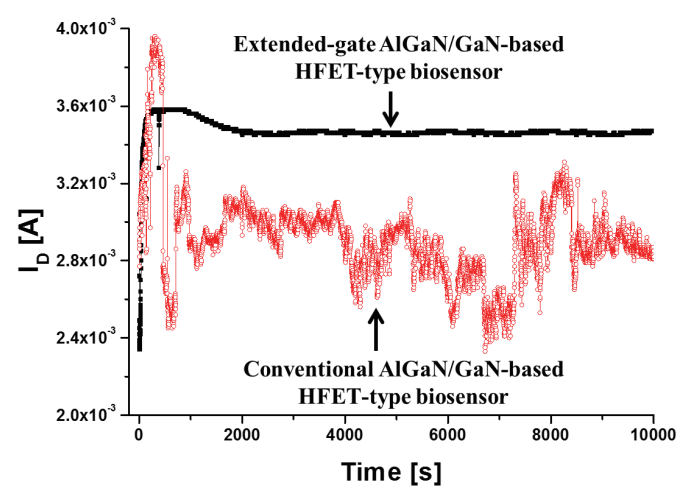

Fig. 7. (Color online) Comparison of the long-term stability of the extended-gate AlGaN/GaNbased HFET-type biosensor with that of the conventional AlGaN/GaN-based HFET-type biosensor.

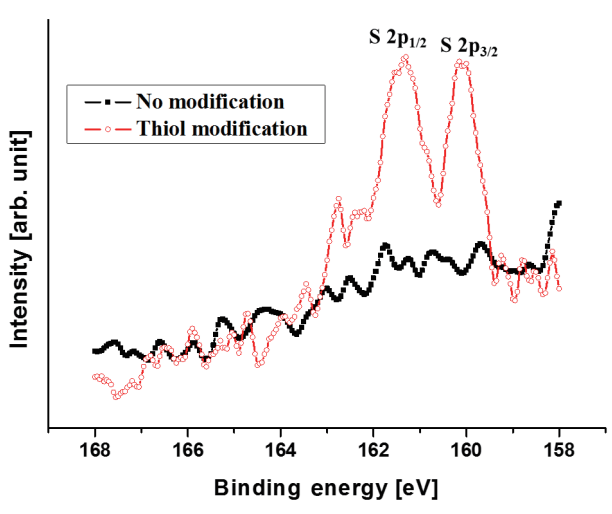

(a)

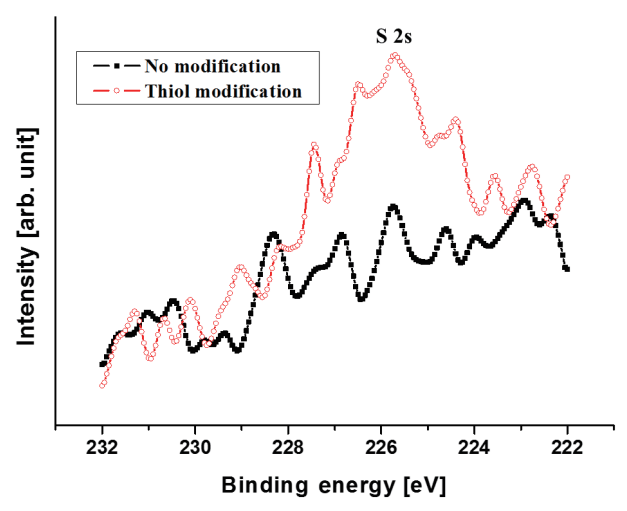

(b)

Fig. 8. (Color online) (a) S 2p and (b) S 2s high-resolution XPS spectra before and after SAM modification of the Au-coated GaN sample.

\section{Conclusions}

The extended-gate AlGaN/GaN-based HFET-type biosensor was fabricated and its characteristics were investigated. Au, which exhibits chemical affinity to thiol, was used as the gate metal in order to immobilize the SAM. An extended-gate AlGaN/GaN-based biosensor employing a commercial $\mathrm{Ag} / \mathrm{AgCl}$ reference electrode for the detection of streptavidin-biotin complexes was investigated. The drain current decreased because of the negative charges produced when thiol was injected into the solution and immobilized on the Au sensing gate. The drain current decreased when streptavidin was immobilized 
owing to the net negative charges of the streptavidin. Finally, biotin was injected in order to detect streptavidin-biotin complexes. The drain current decreased owing to the binding of biotin. Biotin concentrations of 1000 to $0.1 \mathrm{ng} / \mathrm{ml}$ were used as the detection target to evaluate the characteristics of the biosensor. Our XPS measurements verified the SAM formation on the Au surface. In addition, the long-term stability of the biosensor was demonstrated in the PBS solution.

From these results, we confirmed that streptavidin-biotin complexes can be detected by measuring the variation in the drain current of extended-gate $\mathrm{AlGaN} / \mathrm{GaN}$-based HFET-type biosensors.

\section{Acknowledgements}

This study was supported by the BK21 Plus Project funded by the Ministry of Education, Korea (21A20131600011).

\section{References}

1 D. G. Wang, J. B. Fan, C. J. Siao, A. Berno, P. Berno, P. Young, R. Sapolsky, G. Ghandour, N. Perkins, E. Winchester, J. Spencer, L. Kruglyak, L. Stein, L. Hsie, T. Topaloglou, E. Hubbell, E. Robinson, M. Mittmann, M. S. Morris, N. Shen, D. Kilburn, J. Rioux, C. Nusbaum, S. Rozen, T. J. Hudson, R. Lipshutz, M. Chee and E. S. Lander: Science 280 (1998) 1077.

2 P. Brown and D. Botstein: Nat. Gen. 21 (1999) 33.

3 K. E. Nelson, L. S. Gamble, L. S. Jung and M. S. Boeckl: Langmuir 17 (2001) 2807.

4 D.-S. Kim, Y.-T. Jeong, H.-J. Park, J.-K. Shin, P. Choi, J.-H. Lee and G. Lim: Biosens. Bioelectron. 20 (2004) 69.

5 C. Yuan, A. Chen, P. Kolb and V. T. Moy: Biochemistry 39 (2000) 10219.

6 B. S. Kang, H. T. Wang, F. Ren and S. J. Pearton: J. Appl. Phys. 104 (2008) 031101.

7 B. S. Kang, J. J. Chen, F. Ren, S. J. Pearton, J. W. Johnson, P. Rajagopal, J. C. Roberts, E. L. Piner and K. J. Linthicum: Appl. Phys. Lett. 89 (2006) 122102.

8 B. S. Kang, F. Ren, L. Wang, C. Lofton, W. W. Tan, S. J. Pearton, A. Dabiran, A. Osinsky and P. P. Chow: Appl. Phys. Lett. 87 (2005) 023508.

9 J. Choi, H. H. Lee, J. Ahn, S.-H. Seo and J.-K. Shin: Jpn. J. Appl. Phys. 51 (2012) 06FG05.

10 I. Kwon, H.-H. Lee, J. Choi, J.-K. Shin, S.-H. Seo, S.-W. Choi and H.-S. Chun: Jpn. J. Appl. Phys. 50 (2011) 06GL08.

11 D.-S. Kim, J.-E. Park, J.-K. Shin, P. K. Kim, G. Lim and S. Shoji: Sens. Actuators, B 117 (2006) 488.

12 D.-K. Kim, V. Sinduri, Y.-W. Jo, D.-S. Kim, H.-S. Kang, J.-H. Lee, Y. S. Lee, Y. Bae, S.-H. Hahm and J.-H. Lee: Solid-State Electron. 100 (2014) 11.

13 D. Y. Petrovykh, H. Kimura-Suda, L. J. Whitman and M. J. Tarlov: J. Am. Chem. Soc. 125 (2003) 5219.

14 Y. Wang and W. Lu: Phys. Status Solidi A 208 (2011) 1623.

15 M. J. Schoning and A. Poghossian: Analyst 127 (2002) 1137.

16 Y. Wang and W. Lu: Phys. Status Solidi A 208 (2011) 1623.

17 R. Thapa, S. Alur, K. Kim, F. Tong, Y. Sharma, M. Kim, C. Ahyi, J. Dai, J. W. Hong, M. Bozack, J. Williams, A. Son, A. Dabiran and M. Park: Appl. Phys. Lett. 100 (2012) 232109.

18 D.-S. Kim, H.-J. Park, J.-E. Park, J.-K. Shin, S.-W. Kang, H.-I. Seo and G. Lim: Sens. Mater. 17 (2005) 259. 